\title{
On the Number of k-Crossing Partitions
}

\author{
Benedict Irwin ${ }^{1}$ \\ ${ }^{1}$ University of Cambridge
}

May 5, 2021

\section{Abstract}

I introduce $k$-crossing paths and partitions and count the number of paths for each number of desired crossings $k$ for systems with 11 points or less. I give some conjectures into the number of possible paths for certain numbers of crossings as a function of the number of points.

\section{Introduction}

A order $n$ meandric partition is a set of the integers $1 \cdots n$, such that a path from the south-west can weave through $n$ points labeled $1 \cdots n$ without intersecting itself and finally heads east (examples are shown in Fig. 1). Counting the number of possible paths for $n$ points is a tricky problem, and no recursion relation, generating function or explicit formula for the number of order $n$ meandric partitions appears to have been found. This work is concerned with the number of paths that must intersect themselves exactly $k$ times, where when $k$ is 0 , we have the meandric paths. It is possible to draw a line that deliberately crosses itself as many times as required, because of this we only consider a path to be $k$-crossing if $k$ is the smallest number of crossings possible, that is a path that must cross itself $k$ times (an example of a 3 -crossing path over 9 points is given in Fig. 2).

\section{Results}

Define $a_{k}(n)$ to be the number of configurations of $n$ points where the path through them is forced to cross itself $k$ times. For 0 -crossings on $n$ points we have the open meandric numbers, given in the OEIS as A005316

$$
a_{0}(n)=1,1,1,2,3,8,14,42,81,262,538,1828,3926, \cdots, n=0,1, \cdots
$$


this work has counted this for $k>0$ by calculating all $n$ ! permutations of the $n$ integers and checking to see the minimal number of crossings for each, we then have

\begin{tabular}{|c|c|c|c|c|c|c|c|c|c|}
\hline$n=$ & 0 & 1 & 2 & 3 & 4 & 5 & 6 & 7 & 8 \\
\hline 9 & 10 & $11 \ldots$ & & & & & & & \\
\hline $\begin{array}{c}a_{0}(n)= \\
262\end{array}$ & $\begin{array}{c}1 \\
538\end{array}$ & $\begin{array}{c}1, \\
1828, \cdots\end{array}$ & 1, & 2 & 3 & 8 & 14 & 42 & 81 \\
\hline $\begin{array}{c}a_{1}(n)= \\
2760,\end{array}$ & $\begin{array}{c}0 \\
6604\end{array}$ & $\begin{array}{c}0, \\
25176, \ldots\end{array}$ & 1, & 4 & 10 & 36 & 85 & 312 & 737 \\
\hline $\begin{array}{c}a_{2}(n)= \\
10490,\end{array}$ & $\begin{array}{c}0, \\
30842,\end{array}$ & $\begin{array}{c}0, \\
131676, \cdots\end{array}$ & 0 & 0 & 8 & 42 & 168 & 760 & 2418 \\
\hline $\begin{array}{c}a_{3}(n)= \\
22240\end{array}$ & $\begin{array}{c}0, \\
83066,\end{array}$ & $\begin{array}{c}0, \\
398132, \cdots\end{array}$ & 0 & 0 & 2 & 16 & 164 & 944 & 4386 \\
\hline $\begin{array}{c}a_{4}(n)= \\
37250\end{array}$ & $\begin{array}{c}0, \\
168645,\end{array}$ & $\begin{array}{c}0, \\
908898, \ldots\end{array}$ & 0 & 0 & 1 & 18 & 146 & 1076 & 6255 \\
\hline $\begin{array}{c}a_{5}(n)= \\
51968\end{array}$ & $\begin{array}{c}0 \\
282122,\end{array}$ & $\begin{array}{c}0, \\
1711824, .\end{array}$ & 0 & 0 & 0 & 0 & 96 & 960 & 7388 \\
\hline $\begin{array}{c}a_{6}(n)= \\
55140,\end{array}$ & $\begin{array}{c}0, \\
384065,\end{array}$ & $\begin{array}{c}0, \\
2642444, \cdots\end{array}$ & 0 & 0 & 0 & 0 & 30 & 440 & 6472 \\
\hline $\begin{array}{c}a_{7}(n)= \\
53920\end{array}$ & $\begin{array}{c}0, \\
455944,\end{array}$ & $\begin{array}{c}0, \\
3575040, \ldots\end{array}$ & 0 & 0 & 0 & 0 & 14 & 368 & 5176 \\
\hline $\begin{array}{c}a_{8}(n)= \\
45960,\end{array}$ & $\begin{array}{c}0 \\
484058,\end{array}$ & $\begin{array}{c}0, \\
4336734, \cdots\end{array}$ & 0 & 0 & 0 & 0 & 2, & 66 , & 3542 \\
\hline $\begin{array}{c}a_{9}(n)= \\
32280\end{array}$ & $\begin{array}{c}0, \\
452504,\end{array}$ & $\begin{array}{c}0, \\
4661756, \ldots\end{array}$ & 0 & 0 & 0 & 0 & 1, & 72 & 2011 \\
\hline $\begin{array}{c}a_{10}(n)= \\
25066,\end{array}$ & $\begin{array}{c}0, \\
396493,\end{array}$ & $\begin{array}{c}0, \\
4709856, \ldots\end{array}$ & 0 & 0 & 0 & 0 & 0 & 0 & 1172 \\
\hline $\begin{array}{c}a_{11}(n)= \\
11840,\end{array}$ & $\begin{array}{c}0, \\
309696,\end{array}$ & $\begin{array}{c}0, \\
4291440, \ldots\end{array}$ & 0 & 0 & 0 & 0 & 0, & 0 & 420 \\
\hline $\begin{array}{c}a_{12}(n)= \\
8930,\end{array}$ & $\begin{array}{c}0, \\
225754\end{array}$ & $\begin{array}{c}0, \\
3661348, \ldots\end{array}$ & 0 & 0 & 0 & 0 & 0 & 0 & 201, \\
\hline $\begin{array}{c}a_{13}(n)= \\
2240,\end{array}$ & $\begin{array}{c}0 \\
151849\end{array}$ & $\begin{array}{c}0, \\
2947392, \ldots\end{array}$ & 0 & 0 & 0 & 0 & 0 & 0 & 40 \\
\hline $\begin{array}{c}a_{14}(n)= \\
2040,\end{array}$ & $\begin{array}{c}0, \\
91147\end{array}$ & $\begin{array}{c}0, \\
2103648, \ldots\end{array}$ & 0 & 0 & 0 & 0 & 0 & 0 & 18 \\
\hline $\begin{array}{c}a_{15}(n)= \\
224,\end{array}$ & $\begin{array}{c}0 \\
55030\end{array}$ & $\begin{array}{c}0, \\
1575744, \cdots\end{array}$ & 0 & 0 & 0 & 0 & 0 & 0 & 2 \\
\hline $\begin{array}{c}a_{16}(n)= \\
270,\end{array}$ & $\begin{array}{c}0 \\
26762\end{array}$ & $\begin{array}{c}0, \\
915924, \cdots\end{array}$ & 0 & 0 & 0 & 0 & 0 & 0 & 1 \\
\hline $\begin{array}{c}a_{17}(n)= \\
0\end{array}$ & $\begin{array}{c}0 \\
14627,\end{array}$ & $\begin{array}{c}0, \\
665088, \cdots\end{array}$ & 0 & 0 & 0 & 0 & 0 & 0 & 0 \\
\hline $\begin{array}{c}a_{18}(n)= \\
0,\end{array}$ & $\begin{array}{c}0 \\
5405\end{array}$ & $\begin{array}{c}0, \\
295956,\end{array}$ & 0 & 0 & 0 & 0 & 0 , & 0 & 0 \\
\hline $\begin{array}{c}a_{19}(n)= \\
0,\end{array}$ & $\begin{array}{c}0, \\
2642,\end{array}$ & $\begin{array}{c}0 \\
218508,\end{array}$ & 0 & 0 & 0 & 0 & 0 & 0 & 0 \\
\hline $\begin{array}{c}a_{20}(n)= \\
0,\end{array}$ & $\begin{array}{c}0, \\
641,\end{array}$ & $\begin{array}{c}0, \\
63522, \cdots\end{array}$ & 0 & 0 & 0 & 0 & 0 & 0 & 0 \\
\hline $\begin{array}{c}a_{21}(n)= \\
0,\end{array}$ & $\begin{array}{c}0, \\
293,\end{array}$ & $\begin{array}{c}0, \\
54672, \cdots\end{array}$ & 0 & 0 & 0 & 0 & 0 & 0 & 0 \\
\hline $\begin{array}{c}a_{22}(n)= \\
0,\end{array}$ & $\begin{array}{l}0, \\
48\end{array}$ & $\begin{array}{c}0, \\
8964, \cdots\end{array}$ & 0 & 0 & 0 & 0 & 0 & 0 & 0 \\
\hline $\begin{array}{c}a_{23}(n)= \\
0,\end{array}$ & $\begin{array}{c}0, \\
22,\end{array}$ & $\begin{array}{c}0, \\
9552, \cdots\end{array}$ & 0 & 0 & 0 & 0 & 0 & 0 & 0 \\
\hline $\begin{array}{c}a_{24}(n)= \\
0,\end{array}$ & $\begin{array}{l}0, \\
2,\end{array}$ & $\begin{array}{r}0 \\
706,\end{array}$ & $\begin{array}{r}0 \\
2\end{array}$ & 0 & 0 & 0 & 0 & 0 & 0 \\
\hline $\begin{array}{c}a_{25}(n)= \\
0,\end{array}$ & $\begin{array}{l}0, \\
1,\end{array}$ & $\begin{array}{r}0, \\
972, .\end{array}$ & 0 & 0 & 0 & 0 & 0 & 0 & 0 \\
\hline
\end{tabular}


where the vertical sum over columns of terms gives $n$ !.

\section{Conjectures}

The above information has lead to a few conjectures.

$$
\text { Conjecture 1: } a_{n^{2}}(2 n)=1
$$

this can be converted to words as, there is exactly one path through $2 n$ points that crosses $n^{2}$ times. The partitions associated with these paths are

$$
(4,1,5,2,6,3)
$$

$$
(5,1,6,2,7,3,8,4)
$$

$$
(6,1,7,2,8,3,9,4,10,5)
$$

and a clear interlaced pattern can be seen (an example is given in Fig. 3).

$$
\text { Conjecture 2: } a_{n^{2}-1}(2 n)=2, n>1
$$

Conjecture 3: $a_{n^{2}-2}(2 n)=4 n+2, n>2$

$$
\text { Conjecture 4: } a_{n^{2}-3}(2 n)=8 n+8, n>3
$$

Conjecture 5: $a_{n^{2}}(2 n+1)=2(n+1) 3^{n-1}, n>1$ 


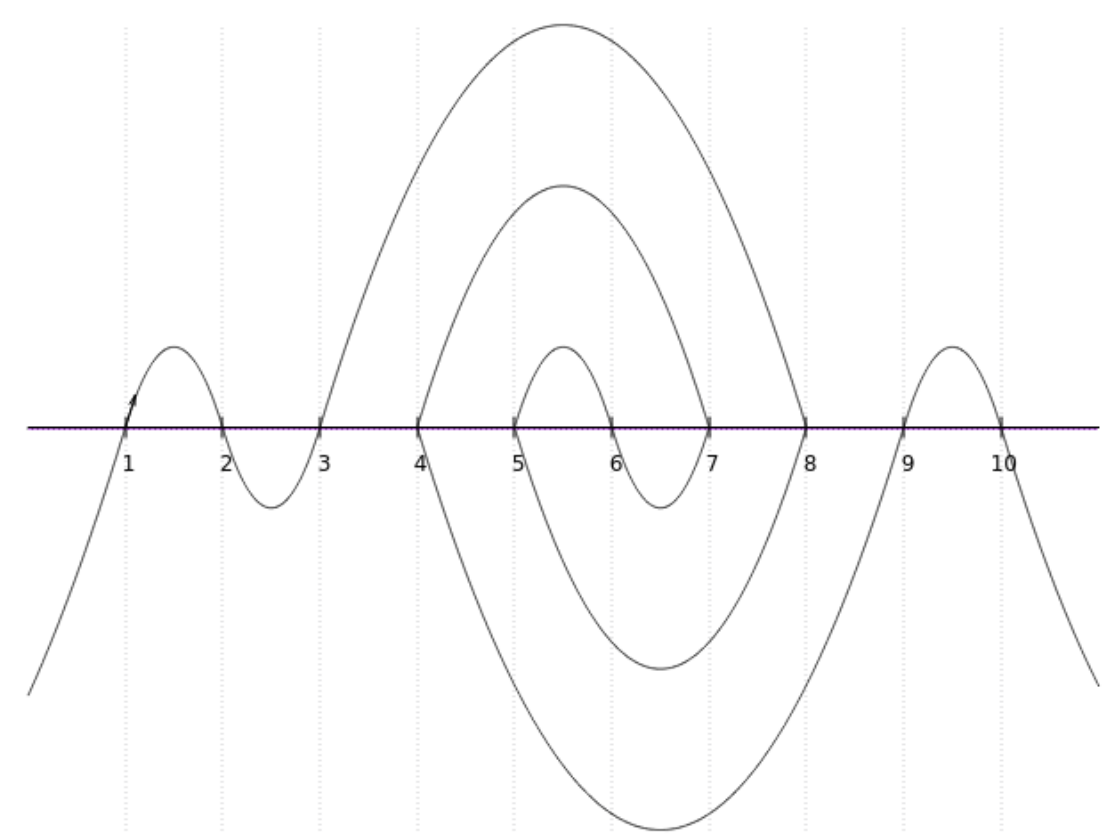

Figure 1: Fig 1. An example of a meandric path (0-crossing path) with $n=10$. This path has meandric partition $(1,2,3,8,5,6,7,4,9,10)$.

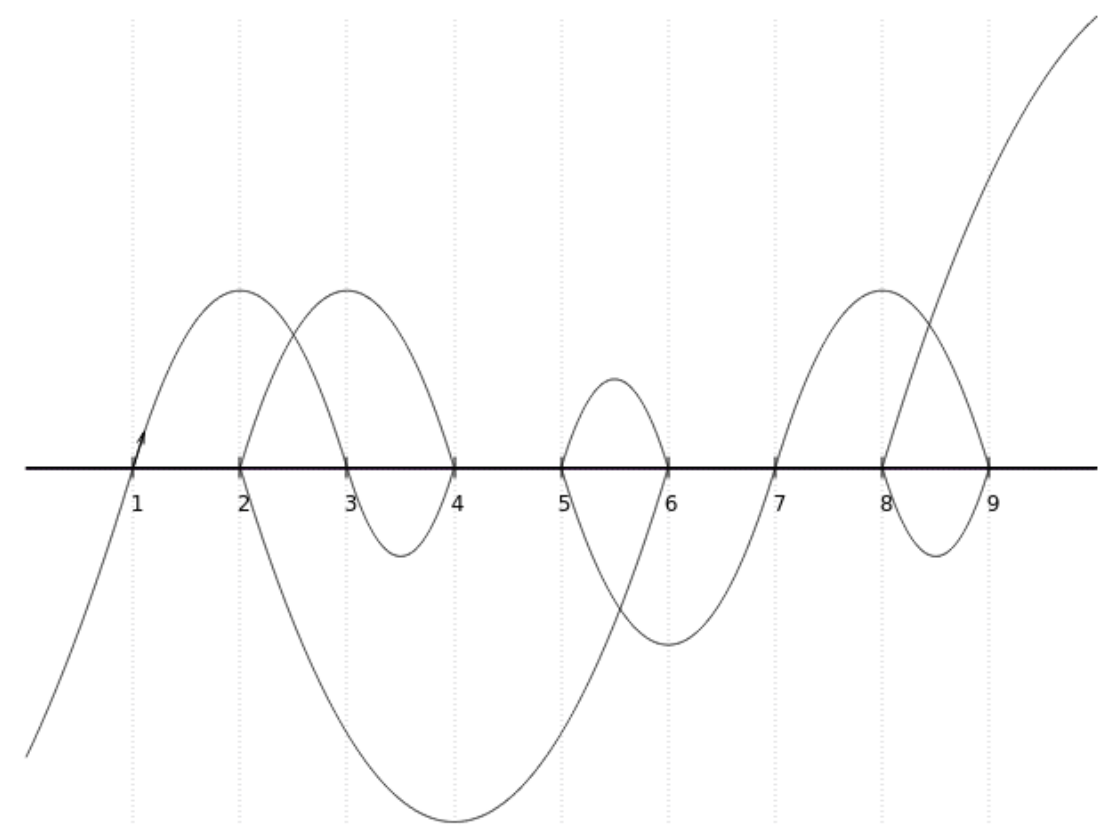

Figure 2: Fig 2. An example of a path that crosses itself 3 times on 9 points. This path has partition $(1,3,4,2,6,5,7,9,8)$. 


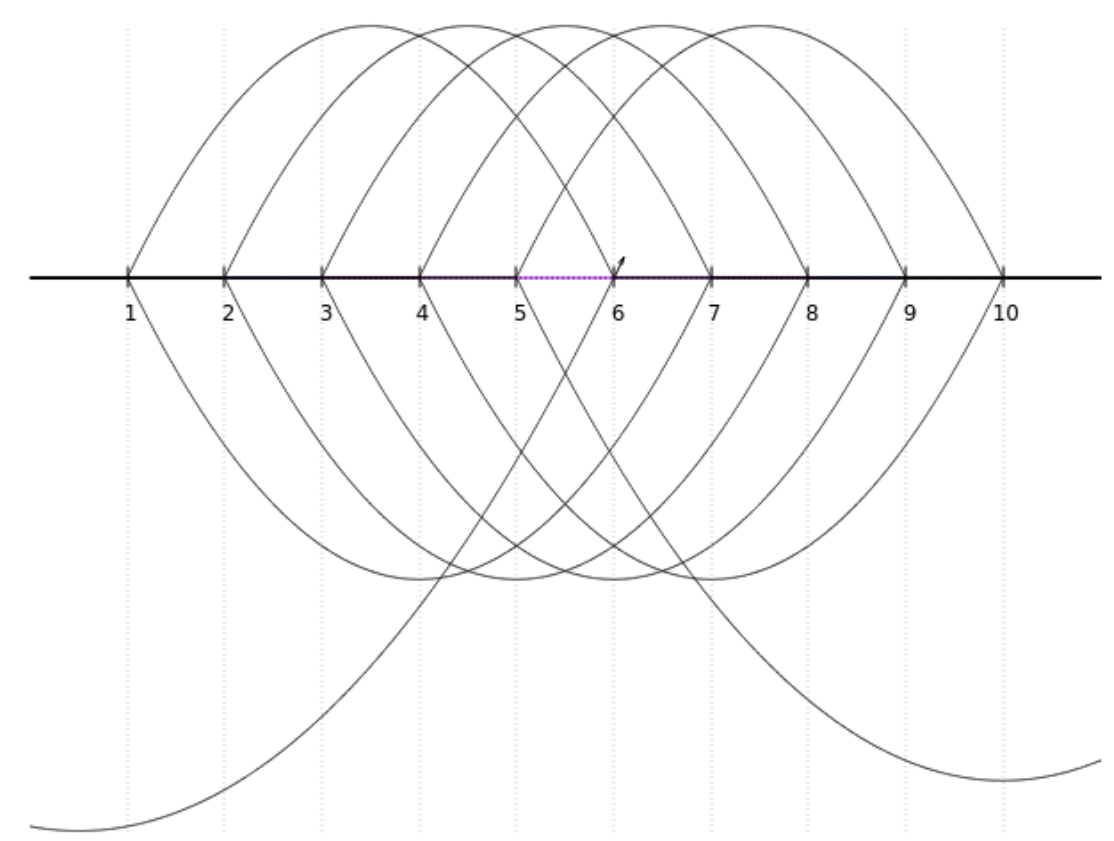

Figure 3: Fig 3. An example of a maximally crossing partition, for 10 points, there is only 1 solution, and Conjecture 1 predicts there is only 1 partition of this type for any even number of points. The number of crossings at the top of the line is a triangular number 10 and the number of crossings at the bottom is the next triangular number 15 . This partition is $(6,1,7,2,8,3,9,4,10,5)$. 